\title{
Correction: Authors' Response to Peer Reviews of "Impact of COVID-19 Testing Strategies and Lockdowns on Disease Management Across Europe, South America, and the United States: Analysis Using Skew-Normal Distributions"
}

Stefano De Leo, PhD

Department of Applied Mathematics, State University of Campinas, Campinas, Brazil

\section{Corresponding Author:}

Stefano De Leo, PhD

Department of Applied Mathematics

State University of Campinas

Rua Sérgio Buarque de Holanda, 651

Campinas, 13083-859

Brazil

Phone: 551935215958

Email: deleo@ime.unicamp.br

\section{Related Article:}

Correction of: https://xmed.jmir.org/2021/2/e28893/

(JMIRx Med 2021;2(2):e29878) doi: 10.2196/29878

In 'Authors' Response to Peer Reviews of 'Impact of COVID-19 Testing Strategies and Lockdowns on Disease Management Across Europe, South America, and the United States: Analysis Using Skew-Normal Distributions"' (JMIRx Med 2021;2(2):e28893) one error was noted.

The reviewer ID of the anonymous reviewer has been removed from the published article to preserve anonymity.
The correction will appear in the online version of the paper on the JMIR Publications website on April 26, 2021, together with the publication of this correction notice. Because this was made after submission to PubMed, PubMed Central, and other full-text repositories, the corrected article has also been resubmitted to those repositories.

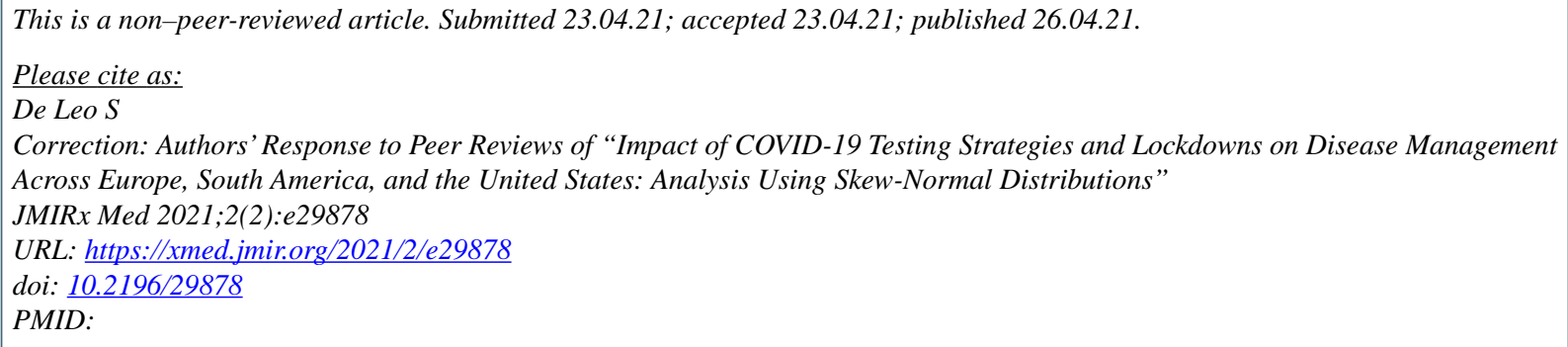

(C)Stefano De Leo. Originally published in JMIRx Med (https://xmed.jmir.org), 26.04.2021. This is an open-access article distributed under the terms of the Creative Commons Attribution License (https://creativecommons.org/licenses/by/4.0/), which permits unrestricted use, distribution, and reproduction in any medium, provided the original work, first published in JMIRx Med, is properly cited. The complete bibliographic information, a link to the original publication on https://xmed.jmir.org/, as well as this copyright and license information must be included. 